\title{
Study on the Stochastic Chance-Constrained Fuzzy Programming Model and Algorithm for Wagon Flow Scheduling in Railway Bureau
}

\author{
Bin Liu \\ Traffic and Transportation School, Lanzhou Jiaotong University, Lanzhou 730070, China \\ Correspondence should be addressed to Bin Liu, liubin0909@mail.lzjtu.cn
}

Received 15 May 2012; Revised 13 August 2012; Accepted 15 August 2012

Academic Editor: Wuhong Wang

Copyright (C) 2012 Bin Liu. This is an open access article distributed under the Creative Commons Attribution License, which permits unrestricted use, distribution, and reproduction in any medium, provided the original work is properly cited.

\begin{abstract}
The wagon flow scheduling plays a very important role in transportation activities in railway bureau. However, it is difficult to implement in the actual decision-making process of wagon flow scheduling that compiled under certain environment, because of the interferences of uncertain information, such as train arrival time, train classify time, train assemble time, and flexible trainsize limitation. Based on existing research results, considering the stochasticity of all kinds of train operation time and fuzziness of train-size limitation of the departure train, aimed at maximizing the satisfaction of departure train-size limitation and minimizing the wagon residence time at railway station, a stochastic chance-constrained fuzzy multiobjective model for flexible wagon flow scheduling problem is established in this paper. Moreover, a hybrid intelligent algorithm based on ant colony optimization (ACO) and genetic algorithm (GA) is also provided to solve this model. Finally, the rationality and effectiveness of the model and algorithm are verified through a numerical example, and the results prove that the accuracy of the train work plan could be improved by the model and algorithm; consequently, it has a good robustness and operability.
\end{abstract}

\section{Introduction}

The train work plan is the core of the daily work plan and the data hub of types of scheduling work in railway bureau. It plays an important role as the whole link between Railway Ministry of China and railway stations and depots. The main purpose of train work plan is to allocate wagons to departure trains. And the wagon flow has to match the time limitation of wagon operations on marshalling stations and the wagon flow direction and train-size limitation. Besides, the train work plan has to meet the demand of wagon loading plan and 
empty wagon reposition plan. All these train operations could be classified as wagon flow scheduling problem. The optimization objective of wagon flow scheduling is to accelerate the rolling stock turnover, to reduce the wagon residence time at railway stations, and to reduce the empty wagon running distance.

For railway wagon flow scheduling problem, many scholars have done a lot of beneficial researches, especially for wagon flow scheduling in railway marshalling stations. Gulbroden studied the railway scheduling in marshalling station by using operations research [1]. Yager and his partner developed an efficient sequencing model for humping in railway marshalling stations [2]. Carey and Carville developed scheduling heuristics analogous to those successfully adopted by train planners using "manual" methods [3]. Lentink et al. discussed how to use network flow method to establish mathematical model to solve train scheduling problem [4]. The robust optimization in railway transportation is discussed by Marton et al. in 2007 [5].

In China, many railway transportation organization methods are fundamentally different from other countries. Wang presented a concept of "price" and used some techniques to transform the wagon flow allocating problem into a transportation problem model in operations research; the objective of the model is to minimize the total price so that the satisfactory solution can be attained by using the calculating method on table [6]. It is worthy of mentioning that the literature [7] is one of the most important literatures in this research field, and many successive studies derive from this. He et al. developed a fuzzy dispatching model for wagon flow scheduling in railway marshalling station and designed a genetic algorithm to obtain the satisfactory solution [8]. And He et al. developed an integrated dispatching model for railway station operations and a computer-aided decision support system [9]. Liu et al. developed a chance-constrained programming model which aimed to reduce the residence time of wagons in the marshalling station and the average delay time of departure trains and designed an improved genetic algorithm to solve the problem [10]. Li et al. addressed the problem of optimizing the marshalling station stage plan with the random train arrival time and developed a dependent-chance programming model and designed a hybrid intelligent algorithm based on stochastic simulation and tabu search [11]. And Li et al. put forwarded a brief survey of stage plans under certain and uncertain environments and with computer-aided dispatching methods and systems. He pointed out the existing and unresolved problems in application of the current theories and methods. What is more, he investigated the direction of future research of railway marshalling stations stage plan [12].

In recent years, with the wide use of Train Dispatch and Management System (TDMS) and Synthetically Automatic Marshalling (SAM) station system in China, the wagon flow information between railway bureau and railway stations are shared completely, so we put forward a new transportation organization concept that is "integralization of railway bureau and railway stations." Thus the wagon flow scheduling in railway bureau can replace the railway station wagon flow scheduling in great extent, and the accuracy of the wagon flow scheduling plan and the overall transportation organization efficiency can be improved dramatically.

Based on above literatures and practical situation in China, considering all kinds of uncertain factors in wagon flow scheduling, such as the stochastic train arrival time and fuzzy train-size limitation of the departure trains, a stochastic chance-constrained fuzzy multiobjective model for the flexible wagon flow scheduling problem is set up. And a hybrid intelligent algorithm based on ant colony optimization (ACO) and genetic algorithm (GA) is also given in this paper. 


\section{Stochastic Chance-Constrained Fuzzy Programming Model}

In this section, we aim at maximizing the satisfaction of departure train-size limitation and minimizing the wagon residence time at railway station to establish the multiobjective optimization model for wagon flow scheduling problem in railway bureau. First, we analyze and formulate the constraints of wagon flow scheduling by considering the some uncertain factor. Then we will summarize and formulate the objective function.

\subsection{Start Time Constraints to Classify a Train}

The train arrival time is stochastic. The lag between train actual and planed arrival time is a random variable with normal distribution, denoted by $\varepsilon_{i}$. Let $T_{i}^{\prime \prime}$ be the planed arrival time of train $i$, so the actual arrival time of train $i$ is $T_{i}^{\prime}=T_{i}^{\prime \prime}+\varepsilon_{i}, T_{i}$ the earliest time after the inspection of train $i, T_{i}=T_{i}^{\prime}+a_{i}$, where $a_{i}$ is the inspection time of train $i$. Assume that a train classifying process is in a time segment $k$. Let $t_{k}$ be the start time to classify a train in the $k$ th time segment, $J_{k}^{i}$ a boolean variable whose value is 1 if train $i$ is classified in the $k$ th time segment, otherwise the value is 0 ; let $n$ be the total number of arriving trains in the stage [8]. So the start time constraints to classify a train are as follows:

$$
\operatorname{Pr}\left(t_{k}-\sum_{i=1}^{n} T_{i} J_{k}^{i} \geq 0\right) \geq \alpha_{1}, \quad k=1,2, \ldots, n
$$

Expression (2.1) denotes that the start time to classify train $i$ in the $k$ th time segment must be after the end time of inspection train $i$. Because of the stochasticity of train arrival time, the end time of train inspection is also stochastic. The expression (2.1) is chance constrained, and the probability of expression (2.1)'s holding is more than or equal to $\alpha_{1}$, where $\alpha_{1}$ is the given confidence level.

\subsection{Start Time Constraints to Assemble a Train}

Assume that a train assemble process is in a time segment $k^{\prime}$. Let $b_{j}$ be the process time of assemble train $j$; it is a random variable with normal distribution. Let $n^{\prime}$ be the total number of departure trains in the stage.

Let $t_{k^{\prime}}^{\prime}$ be the start time to assemble a departure train in the $k$ th time segment, $P_{i k}^{j k^{\prime}}$ be a boolean variable whose value is 1 if train $i$ which is classified in the $k$ th time segment delivers wagons to departure train $j$ which is assembled in the $k^{\prime}$ th time segment, otherwise the value is 0 [7]. So the start time constraints to assemble a departure train are as follows:

$$
\operatorname{Pr}\left(t_{k}+\sum_{i=1}^{n} j_{i} J_{k}^{i}-t_{k^{\prime}}^{\prime} \leq M\left(1-\sum_{i=1}^{n} \sum_{j=1}^{n^{\prime}} P_{i k}^{j k^{\prime}}\right)\right) \geq \alpha_{2}, \quad k=1,2, \ldots, n ; k^{\prime}=1,2, \ldots, n^{\prime}
$$

where $j_{i}$ is the random classifying time of train $i$ with normal distribution. And $M$ is an extremely big positive number. Expression (2.2) denotes that the start time to assemble train $j$ in the $k^{\prime}$ th time segment must be after the end time to classify train $i$ if inbound train $i$ 
delivers wagons to departure train $j$. And the probability of expression (2.2)'s holding is more than or equal to $\alpha_{2}$, where $\alpha_{2}$ is the given confidence level.

\subsection{Wagon Flow Delivers Relationship Constraints}

Let $m_{i r}$ be the arrival wagon number in classified train $i$ whose destination is direction $r$. And $v_{j}$ is the train-size limitation of departure train $j, q$ is total number of destination direction in arrival train, $x_{i r}^{j}$ is decision variable which means the departure train $j$ wagon number whose destination direction is $r$ and from arrival train $i$, and $\Omega(j)$ is the total destination direction number of departure train $j . Q_{j}$ is a boolean variable, its value is 1 if the $j$ th scheduled departure line is occupied by the train, otherwise the value is 0 [7]. So wagon flow delivers relationship constraints that are as follows:

$$
\begin{gathered}
\sum_{j=1}^{n^{\prime}} x_{i r}^{j}=m_{i r}, \quad i=1,2, \ldots, n ; r=1,2, \ldots q, \\
\sum_{i=1}^{n} \sum_{r=1}^{q} x_{i r}^{j}=v_{j} \bullet Q_{j}, \quad j=1,2, \ldots, n^{\prime} .
\end{gathered}
$$

Expression (2.3) denotes that the wagon to direction $r$ from arrival train $i$ can be delivered to different departure train with the same direction. Expression (2.4) denotes the departure train-size limitation which will be further discussed in Section 2.6.

\subsection{Train Departure Time Constraints}

Let $d_{j}^{\prime}$ be the scheduled departure time of train $j$ in train timetable, $h_{j}$ the inspection time of departure train $j, z$ the convoy time needed of the departure train from classification yard to departure yard, $d_{j}$ the latest time of the assembling of train $j$ that should be completed, $d_{j}=d_{j}^{\prime}-h_{j}-z, B_{k^{\prime}}^{j}$ a boolean variable whose value is 1 if train $j$ is assembled in the $k$ th time segment, otherwise the value is 0 , and $d_{j}^{*}$ the lag between train $j$ scheduled departure time and actual departure time [9]. So train departure time constraints are as follows:

$$
\operatorname{Pr}\left(t_{k^{\prime}}^{\prime}+b_{j} B_{k^{\prime}}^{j}-d_{j} \leq M\left(1-B_{k^{\prime}}^{j}\right)\right) \geq \alpha_{3}, \quad k^{\prime}=1,2, \ldots, n^{\prime} ; j=1,2, \ldots, n^{\prime} .
$$

Expression (2.5) denotes that the completed time to assemble train $j$ must not exceed the latest time that is determined by the train timetable so that train $j$ will depart on time. As the classifying time of a train is stochastic, the probability of expression (2.5)'s holding is more than or equal to $\alpha_{3}$, where $\alpha_{3}$ is the given confidence level. And $M$ is an extremely big positive number. 


\subsection{Logic Constraints}

In order to guarantee the logical relationships among variables in the model, the following logic constraints have to conform [8]:

$$
\begin{aligned}
& \sum_{i=1}^{n} J_{k}^{i}=1, \quad k=1,2, \ldots, n, \\
& \sum_{k=1}^{n} J_{k}^{i}=1, \quad i=1,2 \ldots, n .
\end{aligned}
$$

Expression (2.6) denotes only one train can be classified in each time segment; one train can be classified only once:

$$
\begin{aligned}
& \sum_{j=1}^{n^{\prime}} B_{k^{\prime}}^{j}=1, \quad k^{\prime}=1,2 \ldots, n^{\prime}, \\
& \sum_{k^{\prime}=1}^{n^{\prime}} B_{k^{\prime}}^{j}=1, \quad j=1,2 \ldots, n^{\prime} .
\end{aligned}
$$

Expression (2.7) denotes only one train can be assembled in each time segment; one train can be assembled only once:

$$
\sum_{j=1}^{n^{\prime}} \sum_{k^{\prime}=1}^{n^{\prime}} P_{i k}^{j k^{\prime}} \leq M \cdot J_{k^{\prime}}^{i} \quad i=1,2, \ldots, n ; k=1,2, \ldots, n
$$

Expression (2.8) denotes if the arrival train delivers wagons to departure train $j$; train $i$ must be classified, where $M$ is an extremely big positive number:

$$
\sum_{i=1}^{n} \sum_{k=1}^{n} P_{i k}^{j k^{\prime}} \leq M \cdot B_{k^{\prime}}^{j} \quad j=1,2, \ldots, n^{\prime} ; k^{\prime}=1,2 \ldots, n^{\prime} .
$$

Expression (2.9) denotes if the departure train $j$ will be assembled; it must have arrival trains deliver their wagons to the departure train $j$, where $M$ is an extremely big positive number:

$$
\begin{gathered}
J_{k}^{i} \in\{0,1\}, \quad P_{i k}^{j k^{\prime}} \in\{0,1\}, \\
B_{k^{\prime}}^{j} \in\{0,1\}, \quad Q_{j} \in\{0,1\}, \\
i=1,2, \ldots, n, \quad j=1,2, \ldots n^{\prime}, \\
k=1,2, \ldots, n, \quad j=1,2, \ldots n^{\prime} .
\end{gathered}
$$




\subsection{Objective Function}

Let $T_{e}$ be the end time of the wagon flow scheduling stage, then the objective function is as follows [8]:

$$
\max Z_{1}=\sum_{j=1}^{n^{\prime}}\left(\sum_{i=1}^{n} x_{i}^{j}\left(T_{e}-d_{j}^{\prime}-d_{j}^{*}\right)\right)
$$

Let $\lambda_{j}\left(x_{j}\right)$ be the satisfactory function of the actual train size $x_{j}$ compared to the expected train-size $v_{j}$; it is a trapezoidal form fuzzy number as follows [8]:

$$
\lambda_{j}\left(x_{j}\right)= \begin{cases}0, & x_{j}<\underline{v}_{j}^{1} ; \\ \frac{x_{j}-\underline{v}_{j}^{1}}{v_{j}^{1}-\underline{v}_{j}^{1}}, & \underline{v}_{j}^{1} \leq x_{j}<v_{j}^{1} ; \\ 1, & \bar{v}_{j}^{1} \leq x_{j} \leq v_{j}^{2} ; \\ \frac{\bar{v}_{j}^{2}-x_{j}}{\bar{v}_{j}^{2}-v_{j}^{2},} & v_{j}^{2}<x_{j} \leq \bar{v}_{j}^{2} ; \\ 0, & x_{j}>\bar{v}_{j}^{2},\end{cases}
$$

where $\underline{v}_{j}^{1}$ is the minimum number, $\bar{v}_{j}^{2}$ is the maximum number, and $\left[v_{j}^{1}, v_{j}^{2}\right]$ is the expected interval of numbers.

Considering the fuzzy train-size limitation of departure train, we formulated the second objective function:

$$
\max Z_{2}=\sum_{j=1}^{n^{\prime}} \lambda_{j}\left(\sum_{i=1}^{n} x_{i}^{j}\right) .
$$

\section{Chance Constraint Conversion}

For a chance constraint $\operatorname{Pr}\{g(x, \xi) \leq 0\} \geq \alpha$, where $\xi$ is a random variable with distribution function $\Phi$, if function $g(x, \xi)$ has the form $g(x, \xi)=h(x)-\xi$, then $\operatorname{Pr}\{g(x, \xi) \leq 0\} \geq \alpha$ if and only if $h(x) \leq K_{\alpha}$, where $K_{\alpha}=\sup \left\{K \mid K=\Phi^{-1}(1-\alpha)\right\}$. So the deterministic equivalent form of $\operatorname{Pr}\{g(x, \xi) \leq 0\} \geq \alpha$ is as follows [13]:

$$
h(x) \leq K_{\alpha}, \quad K_{\alpha}=\sup \left\{K \mid K=\Phi^{-1}(1-\alpha)\right\} .
$$
[13]:

The same goes for $\operatorname{Pr}\{g(x, \xi) \geq 0\} \geq \alpha$; the deterministic equivalent form is as follows

$$
h(x) \geq K_{\alpha}, \quad K_{\alpha}=\inf \left\{K \mid K=\Phi^{-1}(\alpha)\right\} .
$$


We can convert all the chance constraints in the model above into deterministic equivalent form by expressions (3.1) and (3.2). Let $\Phi_{J}$ be the probability distribution function of $j_{i}, \Phi_{D}$ the probability distribution function of $\varepsilon_{i}$, and $\Phi_{B}$ the probability distribution function of $b_{j}$, and then we have the following deterministic equivalent form.

Equivalence formula for expression (2.1) is

$$
t_{k}-\left(T_{i}^{\prime \prime}+a_{i}+\Phi_{D}^{-1}\left(\alpha_{1}\right)\right) \cdot \sum_{i=1}^{n} J_{k}^{i} \geq 0, \quad k=1,2, \ldots, n
$$

Equivalence formula for expression (2.2) is

$$
M\left(1-\sum_{i=1}^{n} \sum_{j=1}^{n^{\prime}} P_{i k}^{j k^{\prime}}\right)+t_{k}^{\prime}-t_{k}-\Phi_{J}^{-1}\left(\alpha_{2}\right) \cdot \sum_{i=1}^{n} J_{k}^{i} \geq 0, \quad k=1,2, \ldots, n ; k^{\prime}=1,2, \ldots, n^{\prime}
$$

Equivalence formula for expression (2.5) is

$$
M\left(1-B_{k^{\prime}}^{j}\right)-t_{k^{\prime}}^{\prime}-\Phi_{B}^{-1}\left(\alpha_{3}\right) \cdot B_{k^{\prime}}^{j}+d_{j} \geq 0, \quad k^{\prime}=1,2, \ldots, n^{\prime} ; j=1,2, \ldots, n^{\prime} .
$$

\section{A Hybrid Algorithm Based on ACO and GA}

The wagon flow scheduling problem is an NP-complete problem proved by Dahlhaus et al. [14]. In this section, we focus on the hybrid algorithm design based on ant colony optimization (ACO) and genetic algorithm (GA). ACO algorithms are the most successful and widely recognized algorithmic techniques based on ant behaviors, initially proposed by Dorigo in 1992 in his Ph.D. thesis [15]. Genetic algorithms are developed by Holland in 1975. It is a powerful and broadly applicable stochastic search and optimization techniques, inspired by natural evolution, such as inheritance, crossover, mutation, and selection [16]. In this paper, the hybrid algorithm is mainly based on ACO; the crossover and mutation operator of GA is used to avoid the "premature" or "stagnation" of ACO.

Let the arrival train set be $D D$ whose element is $d d_{1}, d d_{2}, \ldots, d d_{m}$ ordered by the train arrival time, and let the departure train set be $C F$ whose element is $c f_{1}, c f_{2}, \ldots, c f_{n}$ ordered by the train departure time in this stage. These two sets are denoted by $D D=$ $\left\{d d_{1}, d d_{2}, \ldots, d d_{m}\right\}$ and $C F=\left\{c f_{1}, c f_{2}, \ldots, c f_{n}\right\}$, respectively. The train makeup destination direction is a set denoted by $\Omega=\left\{r_{1}, r_{2}, \ldots, r_{q}\right\}$ [6].

From Section 2.1, we know that $T_{i}^{\prime}=T_{i}^{\prime \prime}+\varepsilon_{i}, T_{i}=T_{i}^{\prime}+a_{i}$ is the earliest start time to classify train $i$, so the actual classifying time cannot be earlier than $T_{i}$. Let $t_{k}$ be actual start time to classify train $i$ in the time segment $k$. Assume that the departure train sequence is $j_{1}, j_{2} \ldots j_{m^{\prime}}$ in which arrival train can deliver wagons to them and their departure time is $d_{j 1}^{\prime}, d_{j 2}^{\prime}, \ldots d_{j m^{\prime}}^{\prime}$. From the train operating process, it is known that if the end time to classify the arrival train is later than $d_{j n}^{\prime}-h_{j}-z-b_{j}$ (where $1 \leq n \leq m^{\prime}$ ), then the arrival train cannot deliver wagons to the departure train.

Define the classifying time window $\left[E_{i}, D_{i}\right]$ for train $i$, where $E_{i}$ is the earliest start time and $D_{i}$ the latest end time to classify train $i$. Thus the actual classifying time should be between $E_{i}$ and $D_{i}$. Let $\omega_{i}$ be the penalty factor for the delay to classify train $i$, and in this paper $\omega_{i}$ is the wagon number of arrival train $i$. 


\subsection{Initialization}

A classifying sequence of arrival trains can be regarded as an ant's travel path. For example, $(3,1,5, \ldots, i)$ represents the trains classified by the order $3,1,5, \ldots i$, and there are $n$ nodes on the path which represents the arrive trains, respectively. If the $i$ th ant passing node is $j$, it means putting the arrival train $j$ in the position $i$ to classify. In the process of ants travel, the passed nodes make up the train collection and it is the taboo list tabuk, so the every completion of ant's travel makes a new solution.

\subsection{Transition Probability}

Let $L=\left\{\left(i_{1}, i_{2}\right) \mid i_{1}, i_{2} \in D D\right\}$, and we set up a network $G=(D D, L)$, the purpose about this network is to search path that mostly satisfies the constraints of the departure train, such as train-size limitation, punctuality, and inviolate wagon flow direction. At first the pheromone on each edge is equal. And then every ant must make a choice to move to next node; it means that train will be classified in next step. $[15,17]$

Suppose that, at time $t$, the probability of ants $s$ to transfer from train $i_{1}$ to train $i_{2}$ is

$$
P_{i 1, i 2}^{s}(t)= \begin{cases}\frac{\left[\tau_{i 1, i 2}\right]^{\alpha} \cdot\left[\eta_{i 1, i 2}\right]^{\beta}}{\sum_{z \notin \text { tabuk }}\left[\tau_{i 1, z}\right]^{\alpha} \cdot\left[\eta_{i 1, z}\right]^{\beta}}, & i_{2} \notin \text { tabuk } \\ 0, & i_{2} \in \text { tabuk }\end{cases}
$$

in which $\eta_{i 1, i 2}$ is the heuristic information

$$
\eta_{i 1, i 2}=\frac{1}{d_{i}+\xi_{1}\left[\omega_{i} \max \left(0, C_{i}-D_{i}\right)\right]+\xi_{2}\left(\left(D_{i}-E_{i}\right) / b_{j}\right)}
$$

The tabuk is the tabu list that stands for the set of arrival train which has already been classified; $C_{i}$ is the end time for the actual classifying time of train $i ; \xi_{1}$ and $\xi_{2}$ are the weight coefficients; $b_{j}$ is the time of classifying operation; $\alpha$ and $\beta$ are the parameters used to control the relative importance of pheromone and heuristic information.

\subsection{Selection and Local Search Strategy}

Let $q_{0}$ be a constant, $q \in(0,1)$ is a random number, if $q \leq q_{0}$, the next node the ants transfer to is a node that makes $\left[\tau_{i 1, i 2}\right]^{\alpha} \cdot\left[\eta_{i 1, i 2}\right]^{\beta}$ has the maximum value; otherwise, the node will be ensured upon the transition probability by taking the traditional roulette method.

If a local optimal solution is found in the early iteration of ACO, it is easier to appear "premature" or "stagnation" phenomenon, and there is also a need to apply a local search strategy in order to adjust the obviously inappropriate classifying order. So the crossover and mutation strategy of GA is adopted in this paper.

When an ant completes a tour, a train classifying sequence is obtained, then static wagon allocating method is adopted to calculate the "price" of the classifying sequence [6], and then two of the "minimum price" classifying sequences are selected to crossover. 
The crossover strategy [18]: for chromosomes $P_{1}$ and $P_{2}$, randomly generating two random numbers to determine the crossover position, exchange the classifying order between the crossover locations $P_{1}$ and $P_{2}$; if the gene is repeated between and outside of the crossover position, then delete the gene in this location, and then put the lacked gene to the chromosome by ascending order, and then the new chromosomes $P_{1}^{\prime \prime}$ and $P_{2}^{\prime \prime}$ are obtained. Then we calculate $P_{1}^{\prime \prime}$ and $P_{2}^{\prime \prime}$ classifying price and compare to the corresponding price of $P_{1}$ and $P_{2}$. We select the minimum price chromosome $P$ and execute mutation operation, exchange the genes of the two positions which are determined by two random numbers, and calculate the price. Thus the current optimal classifying sequence is represented by the path where the price is the less one of $P$ and $P^{\prime}$.

\subsection{Pheromone Updating Strategy}

The pheromone can be updated as follows:

$$
\tau_{i 1, i 2}(t+1)=(1-\rho) \tau_{i 1, i 2}(t)+\Delta \tau_{i 1, i 2}
$$

where $\rho$ is the parameter to control the pheromone evaporation rate between time $t$ and $t+1$; $1-\rho$ is the retention of the pheromone in the current path. At the beginning, $\tau_{i 1, i 2}=c$ ( $c$ is a constant), and $\Delta \tau_{i 1, i 2}(t+1)$ is the residues pheromone on the passing edge. If the current path is the optimal one, then $\Delta \tau_{i 1, i 2}(t+1)=1 / P^{*}$, where $P^{*}$ is the total price of the optimal sequence; otherwise, $\Delta \tau_{i 1, i 2}(t+1)=0$.

\subsection{The Steps of the Algorithm}

Step 1. Initialization. According to the train arrival information to calculate train the classifying time window, and initialize wagon allocating price table. Set the same amount of pheromone on each edge.

Step 2. Sort trains by their arrival time, and update the wagon allocating price table and calculate the price.

Step 3. Place each ant to each node in $G$, and set tabu list with the corresponding node.

Step 4. Take an ant, calculate the transition probability of selecting the next node to update the tabu list, and then calculate the transition probability, select the node, and update the tabu list again until traverse through all the nodes.

Step 5. Calculate the pheromone that the ant left to each edge, then the ant die.

Step 6. Repeat Steps 3 and 4 until all the ants finish their tour.

Step 7. Calculate the prices of each path that ants choose.

Step 8. Choosing two of the smallest price paths (a path represents a chromosome) $P_{1}$ and $P_{2}$ to compare with path in Step 3, select the less one and make them crossover to obtain new paths $P_{1}^{\prime \prime}$ and $P_{2}^{\prime \prime}$. 
Table 1: Information of arrival trains.

\begin{tabular}{lccccc}
\hline Train code & Arrival time & Train makeup & Train code & Arrival time & Train makeup \\
\hline $\mathrm{dd} 0$ & 0 & $\mathrm{~A} / 15, \mathrm{~B} / 22, \mathrm{C} / 10, \mathrm{D} / 12$ & $\mathrm{dd} 6$ & 112 & $\mathrm{~A} / 24, \mathrm{D} / 22$ \\
$\mathrm{dd} 1$ & 10 & $\mathrm{~A} / 30, \mathrm{~B} / 20$ & $\mathrm{dd} 7$ & 136 & $\mathrm{~A} / 25, \mathrm{C} / 23$ \\
$\mathrm{dd} 2$ & 35 & $\mathrm{~A} / 15, \mathrm{C} / 35$ & $\mathrm{dd} 8$ & 152 & $\mathrm{~B} / 20, \mathrm{C} / 10, \mathrm{D} / 18$ \\
$\mathrm{dd} 3$ & 41 & $\mathrm{~A} / 35, \mathrm{C} / 15$ & $\mathrm{dd} 9$ & 172 & $\mathrm{~A} / 30, \mathrm{C} / 18$ \\
$\mathrm{dd} 4$ & 58 & $\mathrm{~B} / 15, \mathrm{C} / 15, \mathrm{D} / 15$ & $\mathrm{dd} 10$ & 208 & $\mathrm{~B} / 25, \mathrm{C} / 20$ \\
$\mathrm{dd} 5$ & 92 & $\mathrm{~B} / 20, \mathrm{~d} / 25$ & $\mathrm{dd} 11$ & 225 & $\mathrm{~A} / 20, \mathrm{C} / 23$ \\
\hline
\end{tabular}

Step 9. Calculate paths price of $P_{1}^{\prime \prime}$ and $P_{2}^{\prime \prime}$, compare with the prices of $P_{1}$ and $P_{2}$, and select one of the smallest $P$.

Step 10. Execute mutation operation for $P$, and calculate the path price after the mutation compared with the price of $P$, and then select the less one as the optimal path so far.

Step 11. Update the current optimal path, and empty the tabu list tabuk.

Step 12. Judge whether the iterations hit the predetermined number, or whether there is stagnation. If it does, we terminate the algorithm and the output current optimal path; otherwise, go to Step 3, execute the next loop of iteration.

\section{An Illustrative Example}

We take a certain wagon flow scheduling platform as example in one of the railway bureaus in China. Assume that some technological standard operation time is as follows: convoy time is $10 \mathrm{~min}$, arrival inspection time is $35 \mathrm{~min}$, and departure inspection time is $25 \mathrm{~min}$. The lag between train actual and planed arrival time is a normal distribution variable $\mathcal{N}(0,5)$, train classify time obeys $\mathcal{N}(15,3)$, and train assemble time obeys $\mathcal{N}(15,3)$, and departure train-size limitation is a fuzzy trapezoidal variable with parameters of $(40,45,50,52)$.

Since train classify and assemble time conforms $\mathcal{N}\left(\mu, \sigma^{2}\right)$, and the equivalence formula of them can convert to $\inf \left\{K \mid K=\Phi^{-1}(\alpha)\right\}$ by expression $(3.4)$, so $\Phi^{-1}(0.95)=1.6449$ when the confidence level $\alpha=95 \%$.

The arrival train information is shown as in Table 1 . In the convenience of calculating, we set the start time stage is 0 and convert the train arrival time is an integer number which stands for the minutes that train arrival from the stage start time [19]. And assume that there are four train destination directions denoted by A, B, C, and D. The train 0 is a dummy train that represents the wagon flow in the beginning of this stage.

Suppose that all the arrival train can be classified immediately. We can calculate the initial wagon allocating price table according to static wagon allocating problem [6]. In this paper, the Java programming language is used to implement the algorithm above with parameters $\alpha=1, \beta=1, \xi_{1}=1, \xi_{2}=2, q_{0}=0.6, \rho=0.7$. The satisfactory solution of wagon flow scheduling is shown in Table 2 .

From Table 2, we know that all of the departure trains meet the train-size limitation from the point of the fuzzy constraint. And in this stage, there are 495 wagons scheduled to the departure trains. Since we consider the stochasticity and fuzziness in the model and algorithm, the robustness and operability of the work plan of railway bureau is prompted greatly. 
Mathematical Problems in Engineering

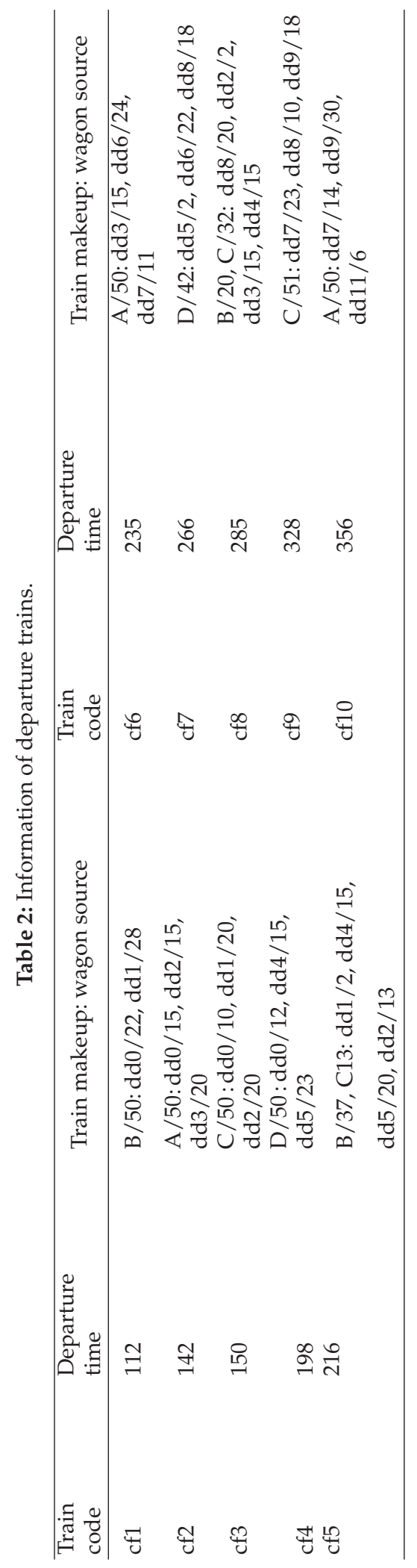




\section{Conclusions}

In this paper, considering the stochasticity of train arrival time, train classify time, and train assemble time and fuzzy train-size limitation, a stochastic chance-constrained fuzzy multiobjective model for wagon flow scheduling is set up based on the uncertain programming theory. By analyzing the model in detail, a hybrid intelligent algorithm based on ACO and GA is given. Furthermore, a numerical example is also offered to verify the rationality and effectiveness of the model and algorithm. As we know, the China railway informatization is very fast in recent years. But the TDMS, SAM, and other management information system are separate and not intelligent in some extent. So it needs to integrate the related systems by optimizing the transportation business models. The model and algorithm proposed in this paper provide the theoretical basis for integrating and optimizing the related systems. We hope the TDMS and SAM will be more practical and intelligent by using our model and algorithm in this paper.

In the future, we will study how to refine the basic wagon flow information, as well as the robust theory for wagon flow scheduling, and how to use synergetic theory in wagon flow and locomotive scheduling.

\section{References}

[1] O. Gulbroden, "Optimal planning of marshalling yard by operation research," ROCEEDINGS of the Symposium on the use of Cybernetics on the Railways, pp. 226-233, 1963.

[2] S. Yager, F. ESaccomanno, and Q. Shi, "An efficient sequencing model for humping in a railway," Transportation Research A, vol. 17, pp. 251-262, 1988.

[3] M. Carey and S. Carville, "Scheduling and platforming trains at busy complex stations," Transportation Research Part A, vol. 37, no. 3, pp. 195-224, 2003.

[4] R. M. Lentink, P. J. Fioole, L. G. Kroon, and C. Woudy, Applying Operations Research Techniques to Planning Train Shunting, John Wiley \& Sons, Hoboken, NJ, USA, 2006.

[5] P. Marton, J. Maue, and M. Nunkesser, "An improved train classification procedure for the hump Yard Lausanne Triage," in Proceedings of the 9th Workshop on Algorithmic Approaches for Transportation Modeling, Optimization, and Systems (ATMOS'09), September 2009.

[6] C. G. Wang, "Study on wagon-flow allocating problem in a marshalling station by using calculating method on-table," Journal of the China Railway Society, vol. 24, no. 4, pp. 1-5, 2002.

[7] S. He, Optimization for railway hub work plan—study on model and algorithm of work plan for railway marshalling station and daily and shift plan for railway hub [Ph.D. thesis], Southwest jiaotong university, 1996.

[8] S. He, R. Song, and S. S. Chaudhry, "Fuzzy dispatching model and genetic algorithms for railyards operations," European Journal of Operational Research, vol. 124, no. 2, pp. 307-331, 2000.

[9] S. He, R. Song, and S. S. Chaudhry, "An integrated dispatching model for rail yards operations," Computers and Operations Research, vol. 30, no. 7, pp. 939-966, 2003.

[10] T. Liu, S. W. He, B. H. Wang, and J. An, "Stochastic chance constrained programming model and solution of marshalling station dispatching plan," Journal of the China Railway Society, vol. 29, no. 4, pp. 12-17, 2007.

[11] H. D. Li, S. W. He, R. Song, and L. Zheng, "Stochastic dependent-chance programming model and algorithm for stage plan of marshalling station," Journal of Transportation Systems Engineering and Information Technology, vol. 10, no. 1, pp. 128-133, 2010.

[12] H. Li, S. He, B. H. Wang et al., "Survey of stage plan for railway marshalling station," Journal of the China Railway Society, vol. 33, no. 8, pp. 13-22, 2011.

[13] B. Liu, Theory and Practice of Uncertain Programming, Physica, Heidelberg, Germany, 2002.

[14] E. Dahlhaus, P. Horak, M. Miller, and J. F. Ryan, "The train marshalling problem," Discrete Applied Mathematics, vol. 103, no. 1-3, pp. 41-54, 2000. 
[15] M. Dorigo and T. Stutzle, Ant Colony Optimization, The MIT Press, Cambridge, Mass, USA, 2004.

[16] M. Gen and R. Cheng, Genetic Algorithms and Engineering Optimization, John Wiley \& Sons, 2000.

[17] W. J. Gutjahr, "A Converging ACO Algorithm for Stochastic Combinatorial Optimization," in Proceedings of the Stochastic Algorithms: Foundations and Applications (SAGA '03), A. Al-Brecht and K. Steinhoefl, Eds., vol. 9 of Springer LNCS 2827, pp. 10-25, 2003.

[18] J. Wu, Research on improved performance of ant colony algorithm by genetic algorithm [M.S. thesis], Taiyuan University of Technology, 2007.

[19] Y. Jing and C. G. Wang, "Model and algorithm of dynamic wagon-flow allocating in a marshalling yard under uncertainty conditions," Journal of the China Railway Society, vol. 32, no. 4, pp. 8-12, 2010. 


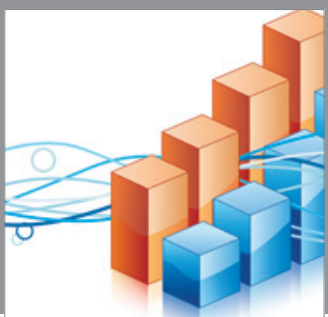

Advances in

Operations Research

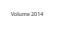

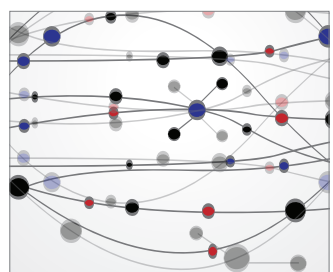

\section{The Scientific} World Journal
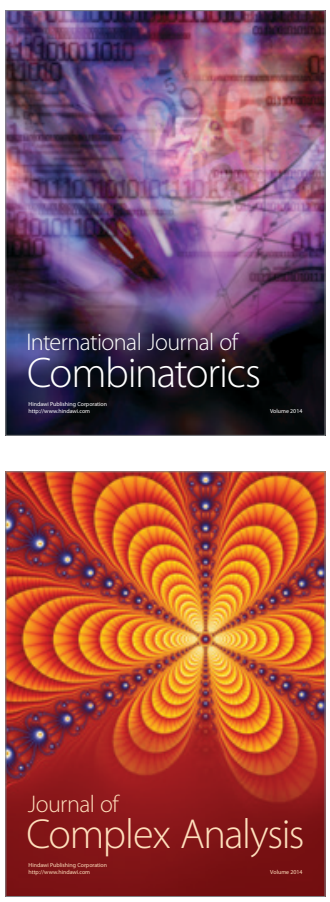

International Journal of

Mathematics and

Mathematical

Sciences
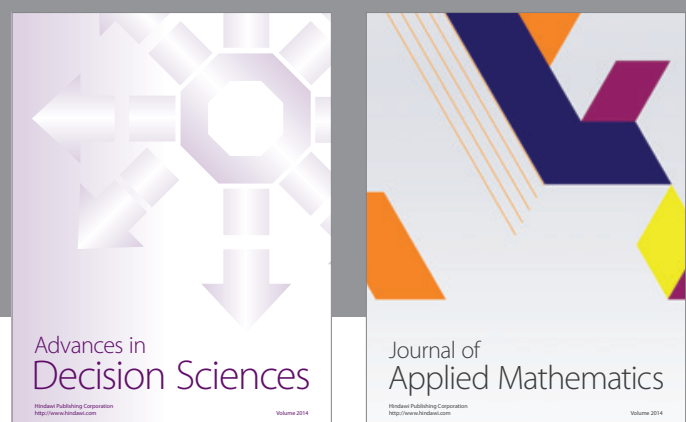

Journal of

Applied Mathematics
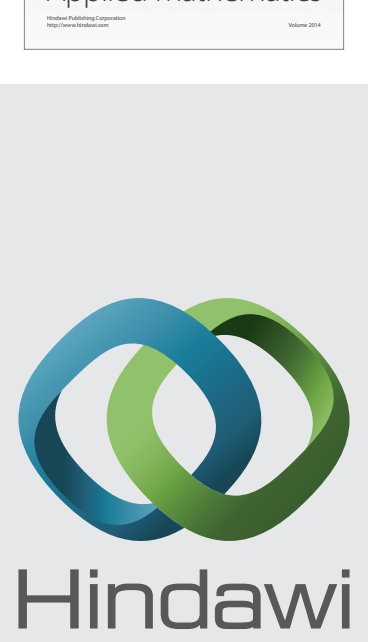

Submit your manuscripts at http://www.hindawi.com
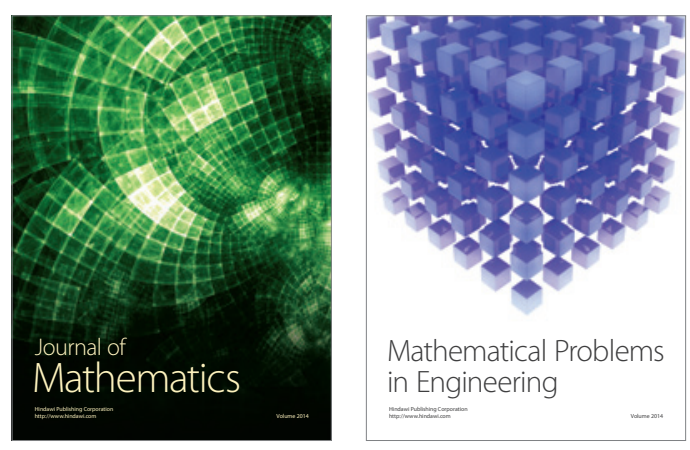

Mathematical Problems in Engineering
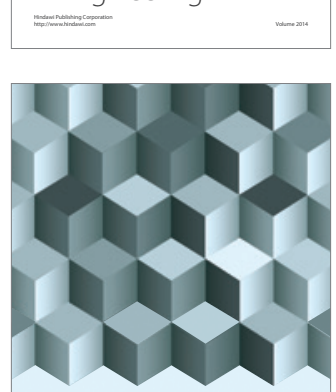

Journal of

Function Spaces
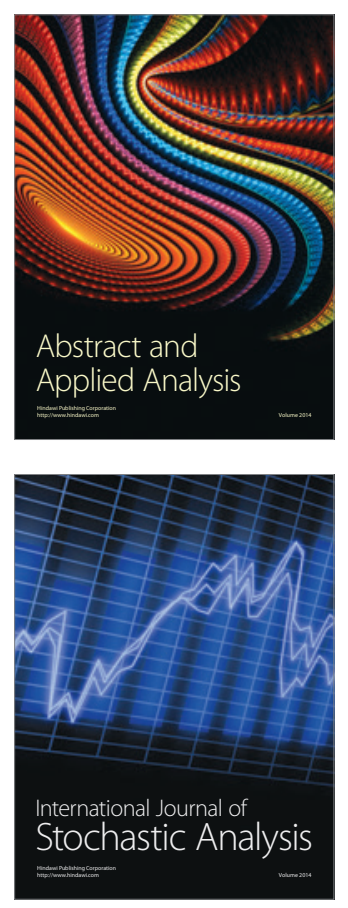

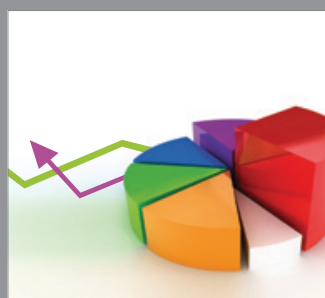

ournal of

Probability and Statistics

Promensencen
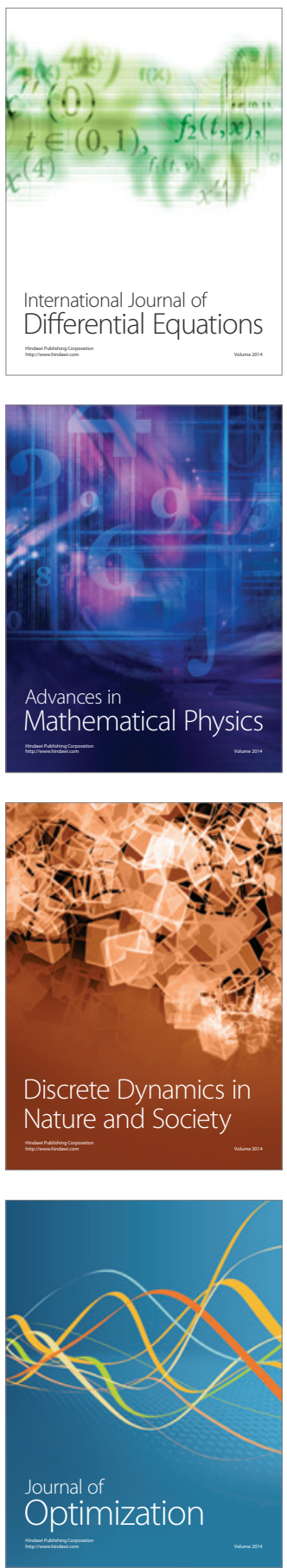